\title{
Merging Fixation for Saliency Detection in a Multilayer Graph
}

\author{
Shiqi $\mathrm{Li}^{\mathrm{a}}$, Cheng Zeng ${ }^{\mathrm{a}}$, Shiping Liu, ${ }^{\mathrm{a}, *}$, Yan $\mathrm{Fu}^{\mathrm{a}}$ \\ ${ }^{a}$ School of Mechanical Science and Engineering, Huazhong University of Science and Technology, Wuhan, 430074, China
}

\begin{abstract}
In this paper, a multilayer graph-based saliency detection algorithm by merging fixation is proposed to effectively detect salient objects in complex scenes. First, the fixation location of an image is acquired by using fixation prediction models. This is based on a motivation that human visual attention system would quickly focus on salient regions before further processing. Then, by merging the fixation saliency map into an over-segmented region map, we can obtain a coarse detection result which most likely contains salient objects. To further improve the performance of our saliency detection, the next key idea is to leverage color contrast between superpixels as features in CIE-Lab space and resolve saliency estimation of coarse regions via a multilayer graph-based framework. The final saliency detection is achieved by combining the coarse detection result with multilayer saliency maps. Extensive experiments are conducted on five benchmark datasets. Experimental results show that the proposed method yields comparable or better results in terms of PR curve, ROC curve, and F-measure, and is robust to deal with both cluttered and clean scenes.
\end{abstract}

Keywords: Fixation prediction, Saliency detection, Multilayer graph, Superpixel.

\section{Introduction}

The notion of saliency generally refers to fixation prediction and salient object detection. Compared with fixation prediction, salient object detection has recently attracted more attention in computer vision community as it arises from the simulation of the human attention mechanism to segment entire salient objects out for further processing. Consequently, the applications of salient object detection have flourished in numerous areas, including recognition [1], [2], image classification [3], [4], and human-robot interaction [5], [6].

Plenty of salient detection methods have been proposed in the past few years, which achieve high performance on simple datasets (the analysis of simple and complex datasets are discussed in section 4.1), and the progress in salient detection is a continuous ascending process. Though much progress has been achieved in detecting salient objects [7], most methods are limited to the case of detecting simple scenes that do not involve complex background. As shown in Fig. 1, when a salient object locates in the low-clutter environment, the state-of-the-art salient detection methods have ideal performance to obtain saliency maps. However, when a scene contains several objects with crowded background, salient methods fail to acquire comparable saliency map compared with ground truth, which is witnessed by the slow progress on complex datasets (see Fig. 5(a)) [7].

Due to the shortcomings of salient methods and the advantage of the human visual attention in high-clutter scenes, it is reasonable to develop saliency detection methods following the studies in psychology and cognition fields of the human vision system. Wang et al. [8], for example, proposed a two-stage approach for saliency detection, which is in accordance with the typical two-stage processing in the human visual system. The first stage is to locate visual pop-outs with extended spectrum residual method [9], and the second stage is to refine the result from the first stage with coherence based propagation. However, using coherence based propagation directly to improve the result from the first stage is not robust in complex scenes. A reasonable solution is the use of a multi-scale superpixel scheme that optimizes the raw result at several different scales [10], [11]. In addition, Mishra et al. [12] utilize the user-specified

${ }^{*}$ Corresponding author

Email address: Shipingliu2015@163.com (Shiping Liu) 


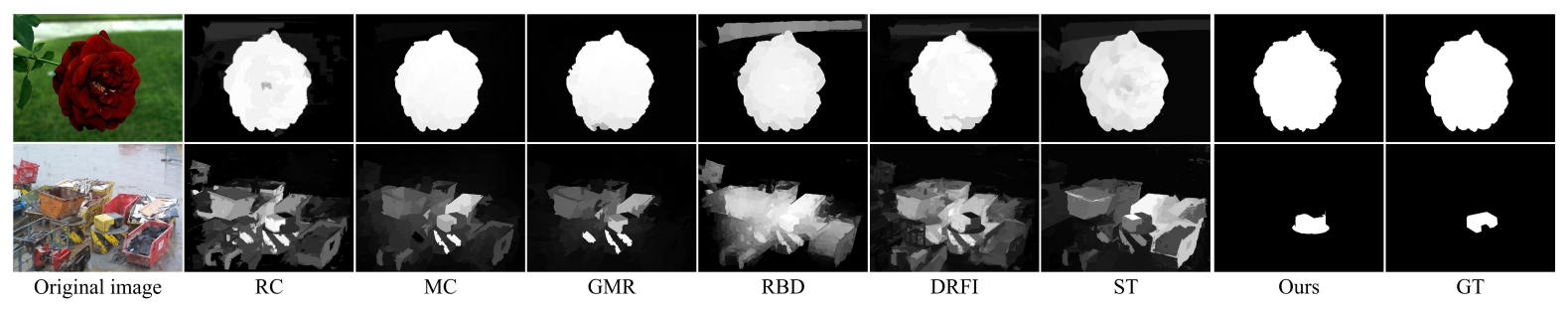

Figure 1: Examples of saliency detection against state-of-the-art methods ([24]-[25], [42], [47]-[49]) in different scenes. All methods highlight the salient object in the clean background. However, only ours succeed in detecting the salient object in complex environments.

fixation points to indicate the salient region in a scene, and then combine monocular cues with stereo and motion features to detect objects. It is obvious that automatically generating fixation points is a better way.

Inspired by two-stage processing in the human visual system, in this paper, we propose a robust two-stage method to automatically detect salient objects in cluttered scenes. First, we apply fixation prediction models to predict visual pop-outs that attract human attention. Combining over-segmented map with fixation prediction map, we can acquire the coarse salient object. Second, due to the superiority of superpixel in reducing computation and multi-scale layers in considering multi-granularity details of crowded scenes, we generate saliency maps in a multilayer superpixel graph-based framework. Finally, we complete the optimization of the coarse result by integrating coarse object and multilayer saliency maps. The pipeline of our method is shown in Fig. 2.

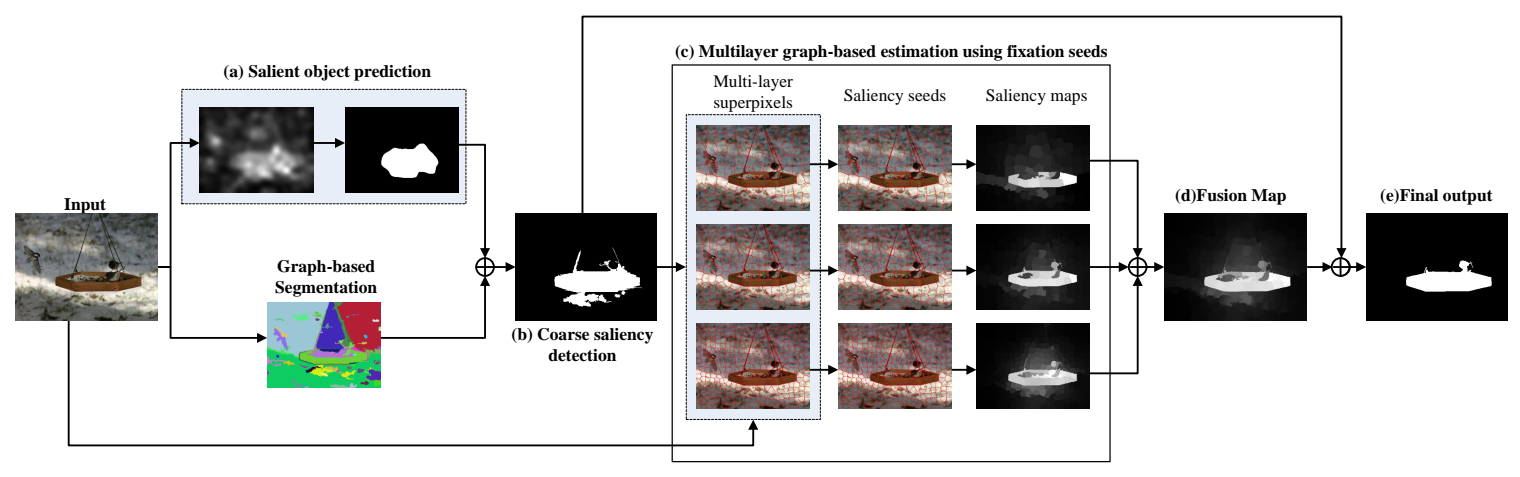

Figure 2: The pipeline of the proposed method. (a): we use fixation models to predict the location of the salient object. (b): Based on the salient position and graph-based segmentation map, the coarse salient object is obtained. (c): Saliency seeds are generated by combining the coarse result with superpixel maps, and then a semi-supervised learning method is used to perform saliency estimation on each layer. (d): Multilayer saliency maps are fused to form the fusion map. (e): the final output is acquired by combining the fused saliency map and coarse salient object.

It is worth mentioning that the work has been proposed by [13], as it shares some common motivation with ours. In their work, they generate the segmentation of object candidates by a generic object proposal method, and then estimate each candidate with fixations via a scoring function proposes. Here, we also take advantage of eye movements to determine coarse salient position but instead of first fully segmenting the scene into object candidates, we merely shallowly segment the scene. Then, we focus on the regions located by fixations. This can potentially lead to better efficiency as the subsequent processes are limited to the coarse salient regions. Another difference lies in that we apply a multilayer superpixel method to optimize the coarse detection result.

The main contributions in this paper are summarized as follows:

1) A fixation-based salient coarse detection method is introduced. The key idea of the method is to measure object saliency by using fixation in crowded environments. Compared with other salient detection methods, the proposed fixation-based method is more robust to locate salient regions in complex scenes. 
2) We propose a multilayer graph-based learning method is proposed to refine coarse result. In particular, we only perform the optimization process of coarse detection regions, which reduces the computational complexity.

The remainder of the paper is organized as follows: Section 2 reviews related works on salient object detection. Section 3 gives the detailed description of the proposed method. Extensive experimental results and comparisons are presented in section 4. Finally, conclusions and future work are drawn in section 5.

\section{Related works}

Broadly speaking, saliency detection methods can be categorized as bottom-up and top-down methods. Bottomup methods are data-driven saliency detection, and top-down methods are task-driven. Comprehensive studies about salient object detection are proposed by [14], [15]. In this work, we concentrate on the following aspects that deal with bottom-up saliency detection.

\subsection{Fixation prediction}

Fixation prediction models compute saliency maps to simulate the real eye movement of the human vision system on scenes. One of the earliest models was proposed by [16]. Their model combined bottom-up visual features of color, intensity, and orientation to construct a saliency map. Hereafter, Bruce et al. [17] proposed a method based on the principle of information maximization sampled from a scene. Hou et al. [9] extracted spectral residual of an image as feature and constructed the corresponding saliency map in the spatial domain. Judd et al. [18] combined different level features with human fixations to learn a saliency map. Borji et al. [19] applied local and global patch rarities in RGB and Lab color spaces for fixation prediction. Zhang et al. [20] utilized the topological structure of Boolean maps computing saliency maps. Erdem et al. [21] used covariance matrices of simple image to integrate different feature dimensions for visual saliency.

\subsection{Superpixel-based saliency detection}

Different from fixation prediction, saliency detection is to highlight the entire salient object. In general, saliency detection can be divided into two categories regarding to processing units, i.e., pixel-based and superpixel-based. Due to the stability and efficiency of superpixels in preserving the structural information of a salient object, superpixelbased methods are growing in popularity. Wang et al. [22] first selected background and foreground seeds successively from compact superpixels, and then integrated background and foreground saliency maps to generate the final saliency map. Jiang et al. [23] constructed a graph-based Markov chain method to compute the connection among superpixels. The same graph-constructed method was also used in [24]. Yang et al. [24] ranked the similarity of superpixels with both foreground and background cues via graph-based manifold ranking. Li et al. [25] used a quadratic programming framework to adaptively optimize the regional saliency values to meet the hypotheses of visual rarity, center-bias, and mutual correlation on a single image. Kim et al. [26] utilized relative location and color contrast of superpixels to perform local saliency estimation, and combined global saliency estimation of high dimensional color space to generate a saliency map. However, the motivation in their work that salient regions and backgrounds can be distinctively separated based on color contrast is hard to meet in high-clutter backgrounds.

\subsection{Multi-scale based saliency detection}

Since superpixel-based detection methods have limited capabilities in detecting complex structure information as indicated in Fig. 1. Recently, a number of methods have been proposed by taking advantage of multi-scale strategies to deal with fine details as well as large-scale structures in saliency detection. Fu et al. [27] first obtained a saliency map by over-segmented algorithm [28] and further performed saliency estimation in an adaptive multi-scale levels merging scheme to consider holistic information of an image. Zhu et al. [10] expected to hold a uniform saliency distribution and implemented saliency estimation by fusing three-scale saliency information. Similar to [10], Yan et al. [29] merged three-level saliency cues by a graphical model to consider small-scale structure information. Qiu et al. [31] generated salient seeds of rare shapes from multi-scale images and resorted to manifold ranking to obtain saliency maps. Most related to [30], we also generate salient seeds at multi-scale layers, but the fixation information we utilized is more robust in cluttered environments. 


\section{The proposed method}

This section details the proposed salient object detection method. First, the coarse salient object detection that combines fixation prediction model and over-segmented algorithm is described in section 3.1. Then, we propose a multilayer superpixel based method to optimize the coarse salient result. An image is segmented into perceptually homogeneous superpixels at different layers for representing different granularity details, which is introduced in section 3.2. On each layer, graph construction and saliency seeds acquisition are discussed in section 3.3. The similarity of superpixels with fixation seeds via graph-based method is ranked in section 3.4. Finally, Section 3.5 integrates multilayer saliency maps into the coarse detection result to finish the optimization process.

\subsection{Coarse salient object detection via fixation points}

The most salient object in a scene is the one that attracts the majority of fixations [31], [13]. Therefore, we take advantage of fixations to obtain a quick estimation of object saliency, and we perform a coarse detection by using graph-based extraction method. In spite of its coarseness, this can potentially lead to better efficiency as we limit our subsequent processes onto the right region.

As described in section 1, fixation prediction map and over-segmented map are first computed. For the former, we use a fixation prediction model to find spatial outliers in scenes that may attract human attention. Generating fixation prediction is the first and vital step for the whole algorithm. Here, we use BMS [20], COV [21], and human fixation for this purpose. We adopt the BMS and COV to provide fixation prediction maps because they are the state-of-theart models for simulating human fixations validated by [7]. Human fixation is used to determine the upper limit of performance for our method. The fixation prediction map is then fed to the following coarse detection. For the latter, we use the popular graph-based superpixel segmentation algorithm proposed by [32] to extract entire salient object boundaries as much as possible, which exhibits good boundary adherence. This algorithm performs an agglomerative clustering of pixels as nodes on a graph, as a result that each superpixel is the minimum spanning tree of the constituent pixels.

Following the aforementioned steps, we obtain a fixation saliency map and an over-partitioned map. The fixation saliency map is first normalized to $(0,1)$ and then thresholded by a fixed $\beta$ to obtain the binary map of the salient object. And the detailed discussion of different thresholds that affect the performance of the proposed method is in section 4.4. Next, all graph-based superpixels that spatially intersect with the nonzero pixels of the binary map are included. As the image boundary is most likely to be background [33], we discard those superpixels that touch the image boundary. The holes inside the eligible superpixels also will be considered as part of the salient object. Finally, the coarse detection of the salient object is accomplished by including candidate superpixels. Fig. 3 depicts the process of coarse detection and shows examples of coarse salient object detection on MSRA-B [34], ECSSD [29], and Judd-A [31] datasets.

After the coarse salient detection, we merely acquire the rough regions of salient objects. As shown in Fig. 3, some rough salient objects contain redundant background, and some lack part of salient objects. In order to retrieve missing regions or discard redundant regions, we use multilayer superpixel semi-supervised learning method to optimize the coarse detection result.

\subsection{Multilayer superpixel extraction and representation}

In order to extract the uniform regions from an image, we adopt superpixel segmentation based methods used by many other saliency detection models. We test five typical algorithms, including Watersheds [35], Normalized cuts [28], Mean shift [36], Turbopixels [37], and SLIC [38] to generate superpixels. We adopt the method mentioned in [38] which uses superpixels to compute color, texture, geometry, and location features. It then trains classifiers to segment 21 object classes and compute multiple parameters to evaluate these algorithms. The experiment shows that Watersheds and Mean shift do not allow the number of superpixels and compactness to be controlled, the edgepreserving of Normalized cuts is very poor, Turbopixels produces the most compact and regular superpixels, whereas it suffers from low computational efficiency. We choose the SLIC for superpixel extraction because of its low computational cost and high segmentation performance.

For a given image, SLIC divides it into approximately equally-sized and perceptually homogeneous superpixels. As mentioned in section 1, we extract superpixels at multi-scale layers, which is achieved by setting different number of superpixels to SLIC. In the proposed method, we increase the number of superpixels in steps of factor 2 between 


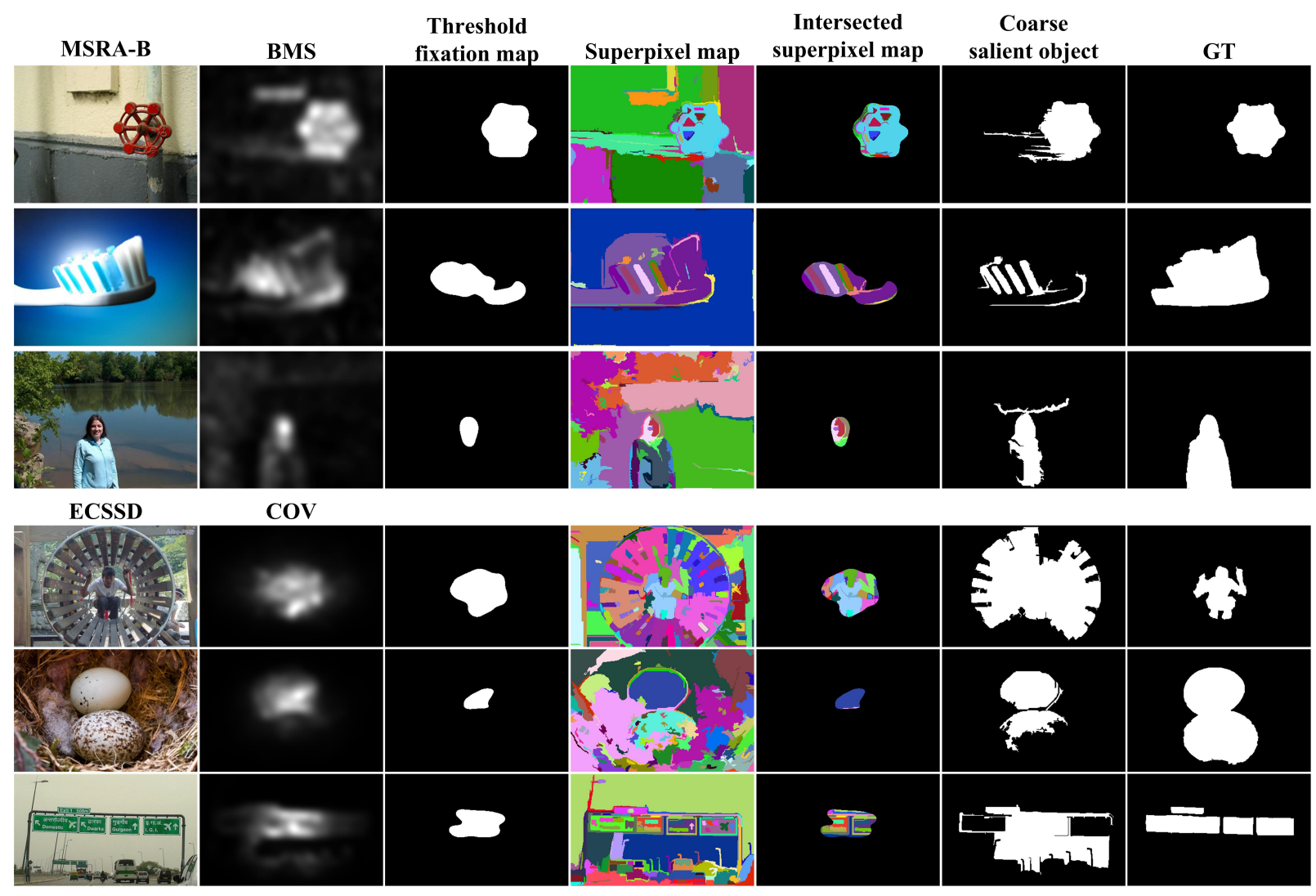

Judd-A

Human

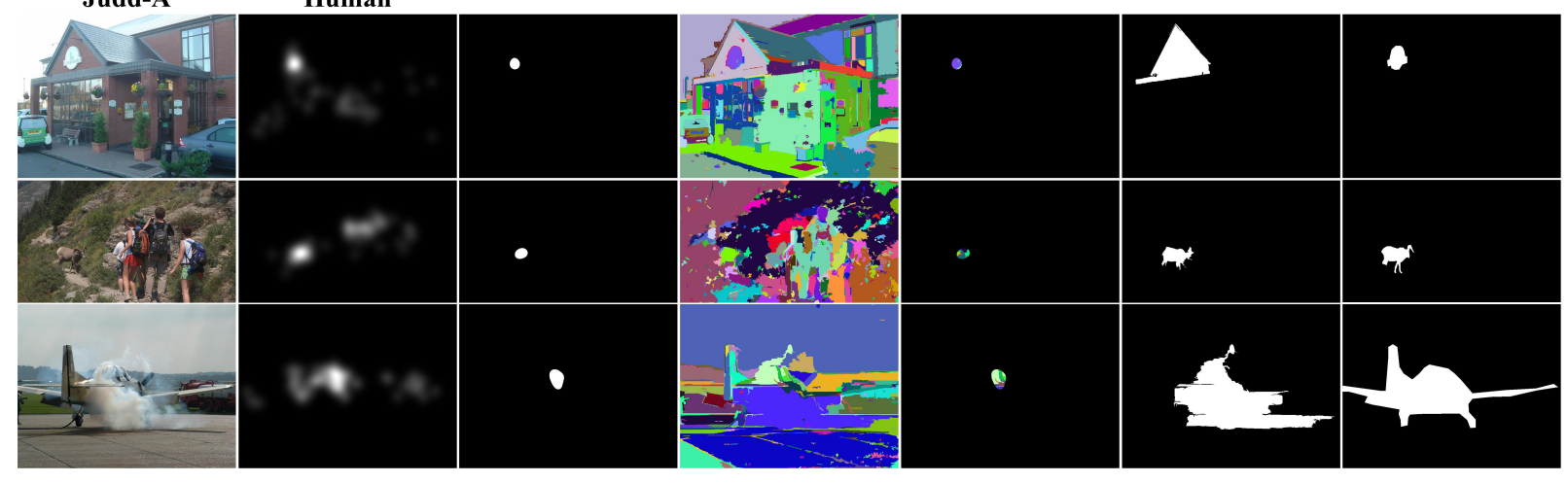

Figure 3: The process of coarse detection on MSRA-B, ECSSD, and Judd-A datasets. Columns from left to right: original image, fixation saliency map (models or human), thresholded saliency map, graph-based superpixel segmentation, intersected superpixel map, ours coarse detection, and ground truth. As shown, the necessary optimization of coarse detection for each dataset from top to bottom: discarding non-salient regions, including salient regions, and both operations to be needed. 

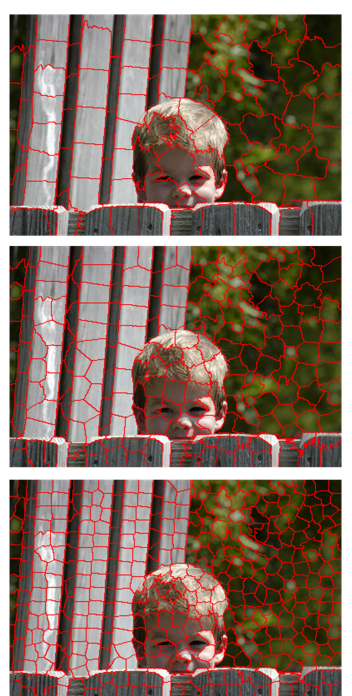

(a)
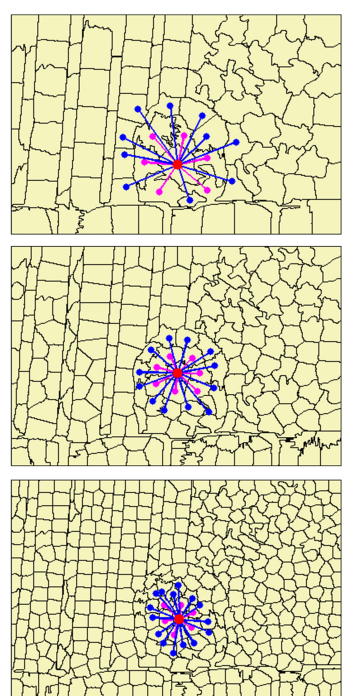

(b)
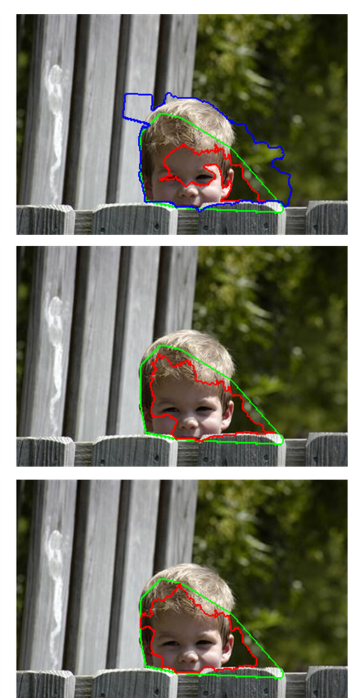

(c)
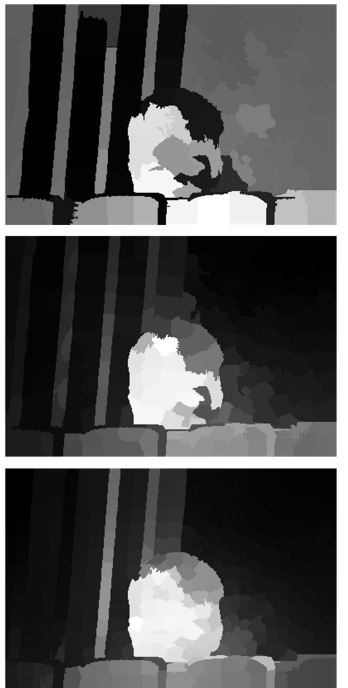

(d)

Figure 4: Illustration of multilayer superpixel extraction, the corresponding connection relationship of superpixels, saliency seeds and saliency maps. From top to down: (a) the number of superpixels increases approximately in steps of factor 2 between two layers. (b) the connection relationship of superpixels corresponding to each scale in (a). The magenta lines represent the direct neighboring superpixels and the blue lines represent the neighbors of those neighbors. (c) the red and green polygons denote saliency seeds and convex hull $C$, respectively. The blue polygon in the coarsest layer indicates the optimization region $R_{c}$. (d) the saliency maps are estimated via graph-based semisupervised learning corresponding to each scale.

scale layers. Fig. 4(a) shows an example of extraction results with different superpixel layers. Since the fewer superpixels, the less possible it is to preserve better visual appearance, we set the coarsest scale to 100 superpixels. For the notations in the following sections, we over-partition an image to build the superpixels set $S^{l}=\left\{s_{1}^{l}, \ldots, s_{N(l)}^{l}\right\}(1$ $=1,2, \ldots, L)$, where $N(l)$ is the number of superpixels on scale $l$. We analyze $L=3$ scale layers in our experimental evaluation.

As Lab performs better to simulate human color perception compared with other color spaces (e.g., RGB), we directly represent features in each superpixel by using the mean color features in CIE-Lab color space.

\subsection{Graph construction and saliency seeds acquisition}

As the image has been segmented into superpixels in the above section, then we build undirected graphs $G^{l}=$ $\left\langle V^{l}, E^{l}\right\rangle$ to reveal the connection relationships of superpixels, where the vertices $V^{l}$ are superpixels and the edges $E^{l}$ are the relevance between $s_{i}^{l}$ and $s_{j}^{l}\left(s_{i}^{l}, s_{i}^{l} \in S^{l}\right)$ in the scale $l$. Like [25], a superpixel connects to both its adjacent superpixels and superpixels sharing common boundaries with its adjacent superpixels, because neighboring superpixels are likely to share similar appearance. The constructed graphs are shown in Fig. 4(b).

Previous works, such as [24] and [30], generate saliency seeds by boundary priors or rare shapes, which are not robust for saliency detection in complex environments. Unlike theirs, we take locations acquired from the coarse detection result as saliency seeds to improve the accuracy of hitting salient objects based on the agreement between fixation and saliency judgments [31]. A shift convex hull $C$ is first constructed based on these superpixels that spatially intersect with the coarse salient object (the green polygon in Fig. 4(c)). We select the superpixels inside the $C$ as saliency seeds. As shown in Fig. 4(c), the area of saliency seeds is increasing with increasing superpixels which can cover more possible salient regions to be queries. In addition, we generate the region to be optimized of the coarse detection result by collecting superpixels that spatially overlap with the convex hull $C$. In order to maximize the probability of covering salient object (i.e., with high recall), we only concentrate on the region $R_{c}$ to be optimized of the coarsest layer (the blue polygon shown in Fig. 4(c)). 


\subsection{Saliency estimation in a graph-based framework}

There is a large family among existing methods for graph-based semi-supervised learning [39]. They are conducted on a graph, where the vertices are labeled and unlabeled samples and the edges reflect the similarities between sample pairs. A function is estimated on the graph based on the prior assumption of consistency. These methods have already been successfully applied in saliency detection due to their effectiveness and efficiency [24][30]. We consider the method proposed in [40]. In section 3.3, we have constructed graphs $G^{l}$ with $V^{l}=\left\{s_{l}^{i} \mid i=1,2, \ldots, N(l)\right\}$ and $E^{l}$ is the weights of edges defined by an affine matrix $W \in R^{N(l) \times N(l)}$ to reveal the similarity between superpixels:

$$
w_{i j}^{l}= \begin{cases}\exp \left(-\left\|s_{i}^{l}-s_{j}^{l}\right\|^{2} / 2 \delta^{2}\right), & \text { if } s_{i}^{l} \text { and } s_{j}^{l} \text { are connected } \\ 0, & \text { otherwise }\end{cases}
$$

where $s_{l}^{i}$ and $s_{l}^{j}$ are the average CIE-Lab color features corresponding to the $i^{\text {th }}$ and $j^{\text {th }}$ superpixels respectively, and $\delta$ is a constant to control the strength of weights. Clearly, the $W$ is a sparse matrix because of the constraints on edges. Then the degree matrix $D$ for the graph $G^{l}$ is:

$$
\left\{\begin{array}{l}
D=\operatorname{diag}\left\{d_{11}, \ldots, d_{N(l) N(l)}\right\} \\
d_{i i}=\sum_{j=1}^{N(l)} w_{i j}, \quad i=1,2, \ldots, N(l)
\end{array}\right.
$$

Given saliency seeds, we need to let every seed iteratively spread its saliency information to its neighbors until a global stable state is achieved. Therefore, a semi-supervised learning method is constructed:

$$
F(t+1)=\alpha P F(t)+(1-\alpha) Y
$$

where $P=D^{-1 / 2} W D^{-1 / 2}$ is the symmetric normalized Laplacian matrix, $F=\left[f_{1}, f_{2}, \ldots, f_{n}\right]^{T}$ indicates the calculated saliency values at time $t, \alpha$ is a trade-off between the neighbors of superpixel and initial information in $(0,1)$, and $Y=\left[y_{1}, y_{2}, \ldots, y_{n}\right]^{T}$ denotes an indication vector, in which $y_{i}=1$ if $s_{i}$ is a saliency seed, and $y_{i}=0$ otherwise. A regularization framework is developed to rewrite (3) by solving the following optimization problem:

$$
F^{*}=\arg \min _{f} \frac{1}{2}\left(\sum_{i, j=1}^{N(l)} w_{i j}\left\|\frac{f_{i}}{\sqrt{d_{i i}}}-\frac{f_{j}}{\sqrt{d_{j j}}}\right\|^{2}+\frac{1-\alpha}{\alpha} \sum_{i=1}^{N(l)}\left\|f_{i}-y_{i}\right\|^{2}\right)
$$

By setting the derivative of $\mathrm{F}$ to be zero, the minimum closed solution can be written as:

$$
F^{*}=(I-\alpha P)^{-1} Y=(D-\alpha W)^{-1} D Y
$$

Given an input image, the saliency of each superpixel on each layer is calculated by (5). Then the saliency maps are obtained by assigning the same saliency value to pixels in the same superpixel. An example of the multilayer saliency maps is shown in Fig. 4(d). As expected, the saliency maps exhibit that an object behaves salient in some but not all scales and multiple levels can capture more salient information.

\subsection{Multilayer combination and final detection output}

On each scale layer $l$, we normalize the saliency map to the range of $(0,1)$ and obtain the combined saliency map $C_{s}$ by averaging three-scale saliency maps. Then, the combined saliency map is used to optimize the coarse detection result. The final optimization process is defined by using a summation:

$$
C_{f}=k C_{d}+(1-k) C_{s}
$$

where $C_{d}$ is the coarse detection map, $C_{f}$ is the combined saliency map, and $k$ is a parameter to control the effect of two maps. We find $k=0.06$ to work well in practice.

After obtaining the final map $C_{f}$, we only deal with the region $R_{c}$ of $C_{f}$ to optimize the coarse detection result. In our work, we use an adaptation of method introduced in [41] to threshold $R_{c}$ which performs an adaptive threshold defined as the mean of $R_{c}$ to acquire the final salient object detection result. 


\section{Experimental results and analysis}

In this section, we exhaustively compare our merging fixation salient (MFS) detection method with twelve stateof-the-art saliency detection methods on five widely used datasets.

\subsection{Experimental setup}

1) Datasets: We evaluate the proposed method on five publicly available datasets: MSRA-B [34], DUT-OMRON [24], ECSSD [29], PASCAL-S [13], and Judd-A [31]. MSRA-B and DUT-OMRON are used to compare methods on a large scale; ECSSD contains semantically meaningful but structurally complex images; PASCAL-S and Judd-A usually contains multiple objects with high-clutter backgrounds. Among these datasets, DUT-OMRON, PASCAL-S, and Judd-A provide salient object annotations and fixations, which can be used in our method to locate salient objects; MSRA-B, and ECSSD only have manually segmented ground truth.

More specifically, MSRA-B contains 5000 images with manually labeled pixel-wise ground truth provided by [42]. Similar to MSRA-B, the 5168 images of DUT-OMRON are also created from more than 140000 natural images, each of which has one or more salient objects and relatively complex backgrounds. As an extension of the Complex Scene Saliency Dataset (CSSD), ECSSD includes 1000 semantically meaningful but structurally complex images, which are obtained from the BSD dataset [43], PASCAL VOC, and the Internet. Judd dataset [18] is the most popular dataset which contains 1003 images for benchmarking fixation prediction models. Because of its popularity, Judd-A [31] selects 900 images from Judd and annotates the most salient objects so that it can be used for benchmarking salient object detection models. In our paper, we choose Judd-A dataset with 900 images to evaluate our method. PASCAL-S is built on the validation set of the PASCAL VOC 2010 segmentation challenge. It contains 850 natural images which are labeled with pixel-wise ground truth.

In order to demonstrate the complexity of scenes on five datasets, we adopt the method proposed by [31] which borrows the idea that the more superpixels a scene contains, the more complex and cluttered it is [44]. To this end, the graph-based superpixel segmentation algorithm is used to segment an image into contiguous regions whose parameters are the same with that of coarse object detection stage. Fig. 5(a) shows the distributions of the number of superpixels on the entire scene. As we can see, the entire scenes in PASCAL-S and Judd-A dataset on average contains more superpixels than scenes in the other datasets. Furthermore, Judd-A dataset is more complex and cluttered than other datasets.

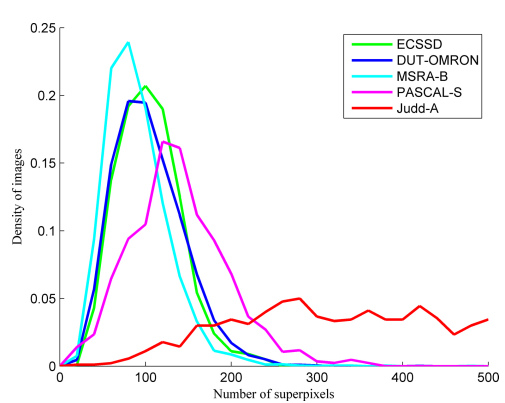

(a)

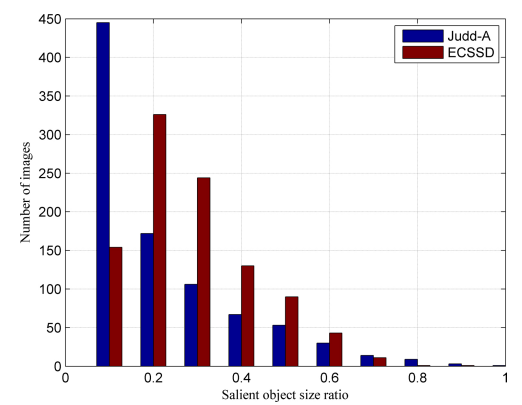

(b)

Figure 5: The statistical information of datasets. (a): distributions of the number of superpixels for the entire scene across five datasets. (b): distribution of salient object size ratio across ECSSD and Judd-A datasets.

2) Implementation details: In the experiments, our method is implemented in MATLAB on an HP workstation with an Intel 17-3720QM 2.6GHz CPU and 8GB RAM. In the coarse stage, the threshold $\beta$ of the fixation saliency map is set to be 0.7. The graph-based superpixel segmentation parameters are smoothing coefficient $\sigma=1$, segmentation coefficient $k=300$, and minimum size $\min =60$. In the optimization stage, we set the control constant $\delta^{2}$ in (1) to be 0.05 , and the scaling factor $\alpha$ in (3) to be 0.99 in graph-based learning, empirically. 


\subsection{Evaluation metrics}

In the experiments, we use three widely adopted metrics for qualitative and quantitative comparison: 1) PrecisionRecall (PR) Curve: The PR curve indicates the capacity of models to retrieve salient objects. For a saliency map, we obtain a binary map $B$ by segmenting it with a fixed threshold $T \in(0,255)$, then precision and recall are calculated with the ground truth $G$ as follows:

$$
\text { Precision }=\frac{B \cap G}{B}, \text { Recall }=\frac{B \cap G}{G}
$$

Varying the threshold T within $(0,255)$, we get 256 pairs of precision and recall. Finally, we average both precision and recall of all images in the dataset to plot the PR curve.

2) F-measure: The F-measure is computed to comprehensively evaluate the performance of models defined as:

$$
F_{\gamma}=\frac{(1+\gamma) \text { Precision } \times \text { Recall }}{\gamma \times \text { Precision }+ \text { Recall }}
$$

where $\gamma$ is set as 0.3 as suggested by [41] to emphasize precision.

3) Receiver Operating Characteristics (ROC) Curve: Similar to PR curve, the ROC curve is the plot of false positive rate $(F P R)$ versus true positive rate (TPR) with fixed thresholds, which is defined as:

$$
T P R=\frac{B \cap G}{G}, F P R=\frac{B \cap G}{\bar{G}}
$$

where $\bar{G}$ denotes the complement of the ground truth $G$. The ROC curve is plotted by varying the fixed threshold from 0 to 255 .

\subsection{Performance evaluation}

We quantitatively and qualitatively compare the proposed method with twelve state-of-the-art saliency detection methods, which include DRFI [42], HDCT [45], RC [46], HC [46], SF [47], MC [23], DSR [11], GMR [24], ST [48], HS [29], RBD [49], GC [50]. In order to fairly evaluate these saliency detection methods, the implementations provided by authors are used to generate saliency maps.

Fig. 6 shows the PR curves, ROC curves, and maximum F-measure by using a fixed threshold of all the compared methods on the five benchmark datasets, respectively. As shown in Fig. 6, our proposed methods work on par with the best methods on all the five datasets. Specifically, for the MSRA-B dataset, the F-measure of our MFS-COV is only $4.3 \%$ less than the best model DRFI. For the DUT-OMRON and ECSSD dataset, the F-measure of MFS-Human and are the best three methods, while the F-measure is $2.7 \%$ and $5.6 \%$ less than the best model DRFI, respectively. Hence, compared with all the compared methods, our methods are only a little worse than the best methods on MSRA-B, DUT-OMRON, and ECSSD. Since MSRA-B, DUT-OMRON, and ECSSD datasets are simple (see the analysis in section 4.1) and not challenging, it is not suitable for showing the advantage of our methods. Moving from simple datasets (e.g., MSRA-B) to more complex datasets (e.g., PASCAL-S and Judd-A), we observe a dramatic degradation in the performance of all methods. The average drop from MSRK-B to Judd-A dataset is $47.2 \%$ over the F-measure (for all the compared methods except ours). Now, one can clearly see that our MFS-Human consistently outperforms all the compared methods on PASCAL-S and Judd-A. In particular, Judd-A focuses on images with different backgrounds, whose salient objects contain diverse sizes and complex structure information. Despite that, our MFS-Human achieves an improvement of $28.5 \%$ in comparison to the second best method DRFI. This is consistent with our motivations that take advantage of fixation and multi-scale superpixel to locate and optimize the salient objects. Designed to meet these objectives, our methods achieve clearly higher performance than the compared methods. In addition, our other two methods (i.e., MFS-COV and MFS-BMS) also achieve higher F-measures than all other methods over Judd-A dataset with cluttered scenes. This indicates that our methods using fixations to locate the most salient object are more robust for different images with simple and complex scenes.

Fig. 10 shows visual comparisons of our methods over several challenging scenes against the other state-of-the-art methods. As can be seen, all the compared methods perform poorly in the scenarios with background clutter (e.g., the tenth and last rows), and our method with human fixation can hit the salient object and detect it. Benefiting from the multi-scale superpixel scheme, our algorithm tends to highlight the most salient regions with different size; therefore, 

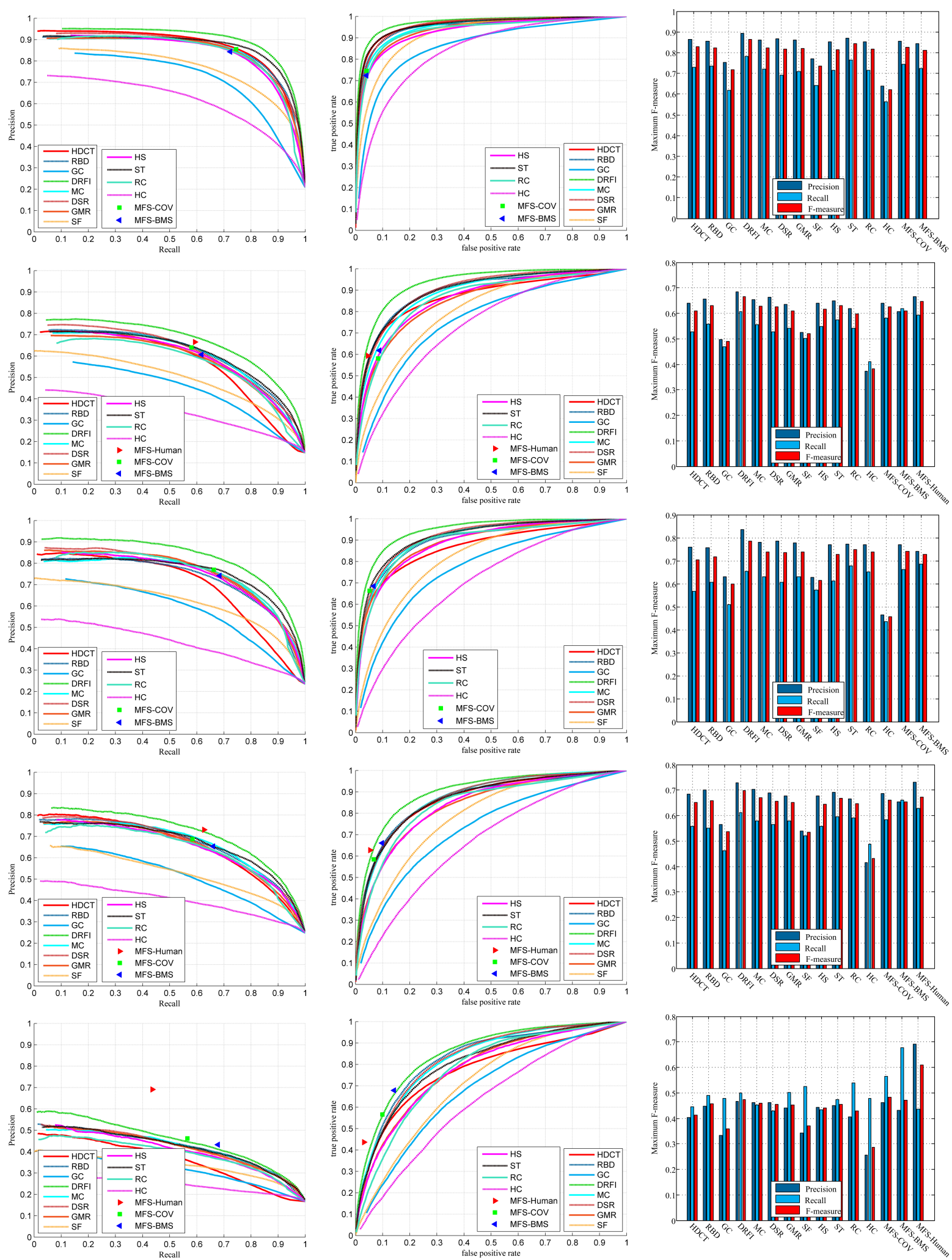

Figure 6: Quantitative Comparison of our methods with state-of-the-art methods on five widely used benchmark datasets from top to down: MSRA-B, DUT-OMRON, ECSSD, PASCAL-S, and Judd-A. (a): PR curves. (b): ROC curves. (c): maximum F-measure of the fixed thresholded saliency map. Our proposed methods are more robust to deal with different scenes. 
our method can not only segment large objects (e.g., the fourth row) but also detect small salient objects which would be suppressed by other methods (e.g., the fifth row). Apparently, the proposed method owns better robustness either in clean backgrounds, or high-cluttered scenes.

\subsection{Components analysis}

We further analyze the effectiveness of different components of our methods: the threshold of the fixation prediction map and the optimization process of the coarse detection stage.

1) Analysis of fixation map thresholding: In the coarse detection stage, we obtain the most salient location by using a fixed threshold $\beta$. In order to study the relationship between fixation threshold and performance in our method, we set the threshold $\beta$ from 0.1 to 0.9 and calculate the corresponding F-measure on ECSSD and Judd-A datasets. As shown in Fig. 7, we observe that both ends of the threshold coordinate lead to poor performance, and the proposed method can perform better by using other fixed thresholds. Meanwhile, it can be seen that the threshold achieving best performance on ECSSD dataset is smaller than that of Judd-A. As shown in Fig. 5(b), the majority of salient objects in Judd-A dataset only occupy less than $10 \%$ of the image, and the opposite holds over the ECSSD dataset. Consequently, a lower threshold could result in a better detection result in ECSSD dataset. In the paper, the reason that we simple set the threshold $\beta$ to a fixed value is because the performance of proposed method is related to the area of salient object, and we can not know the size of salient object in a scene beforehand. We only take into account all different scenes with a certain threshold. In addition, for each threshold $\beta$ on Judd-A dataset, the proposed method with human fixation always achieves better performance than method with fixation prediction models (i.e., BMS and $\mathrm{COV}$ ). This validates that fixation prediction models are still inferior to human in locating salient objects.

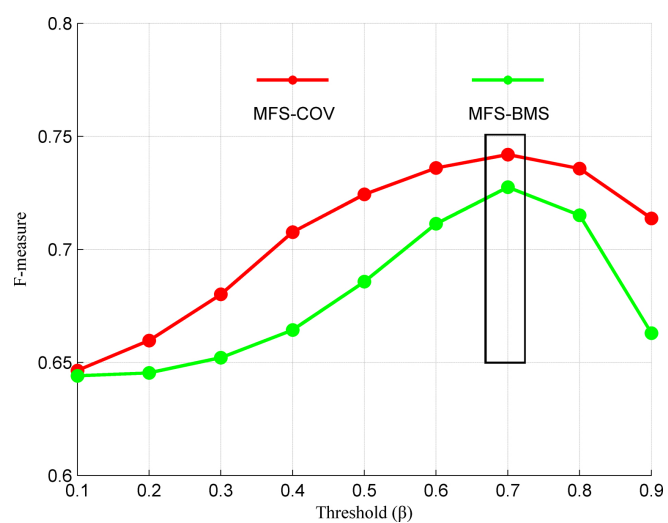

(a) ECSSD

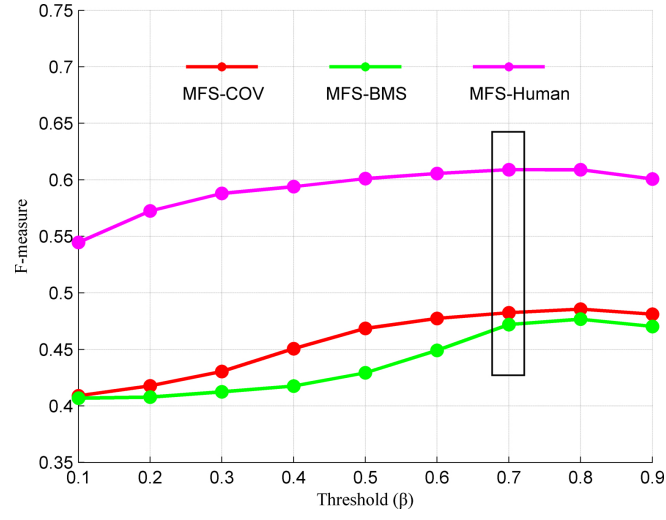

(b) Judd-A

Figure 7: F-measure with the fixation threshold $\beta$ from 0.1 to 0.9 on ECSSD and Judd-A datasets. The black box is the corresponding F-measure in Fig. 6. As can be seen, the proposed method can even achieve higher performance by using other parameters.

2) Analysis of optimization process: To investigate the effectiveness of multilayer graph-based learning, we compare Precision, Recall, and F-measure of the coarse detection stage with that of the optimization stage on PASCAL-S dataset. As shown in Fig. 8, all evaluation metrics grow. Specifically, the F-measure of MFS-BMS increases by $6.7 \%$ from coarse stage to optimization stage, and MFS-COV is 6.9\%. The Precision and Recall of MFS-BMS increase by $8.3 \%, 1.3 \%$, respectively. The MFS-COV increase by $5.2 \%, 11.8 \%$ respectively. Considering that the PASCAL-S dataset is more complex than other datasets except Judd-A (see Fig. 5(a)), the improvement is enough to illustrate the effectiveness of our multilayer learning framework. As can be seen in the examples in Fig. 8, the coarse detection results either include non-salient regions or lack salient regions, and our methods correctly include/discard superpixels. 

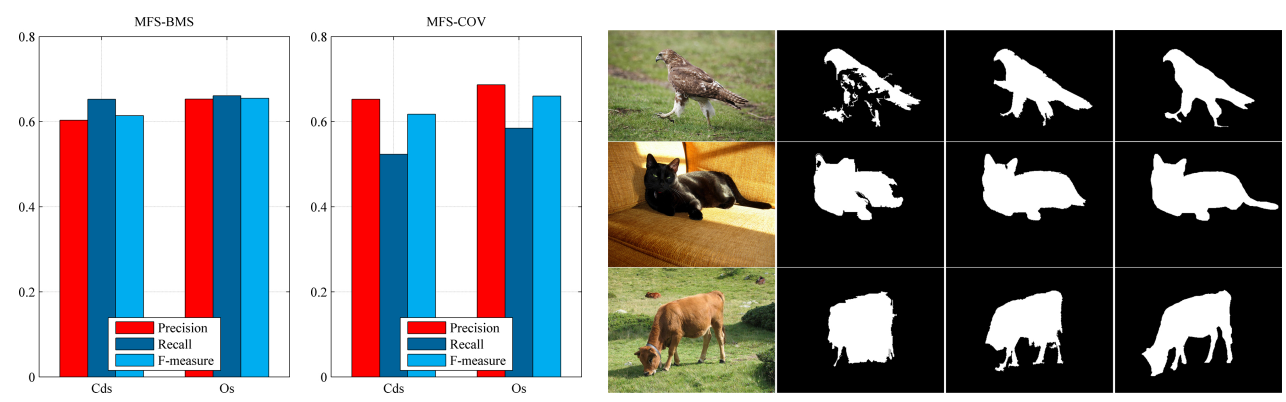

Figure 8: Comparisons of coarse detection stage (Cds) and optimization stage (Os) on PASCAL-S dataset. Left: comparisons of Precision, Recall, and F-measure between two stages of our two methods (i.e., MFS-COV and MFSBMS). Right: examples of the optimization process; from left to right: original image, coarse detection, final detection result, and ground truth.

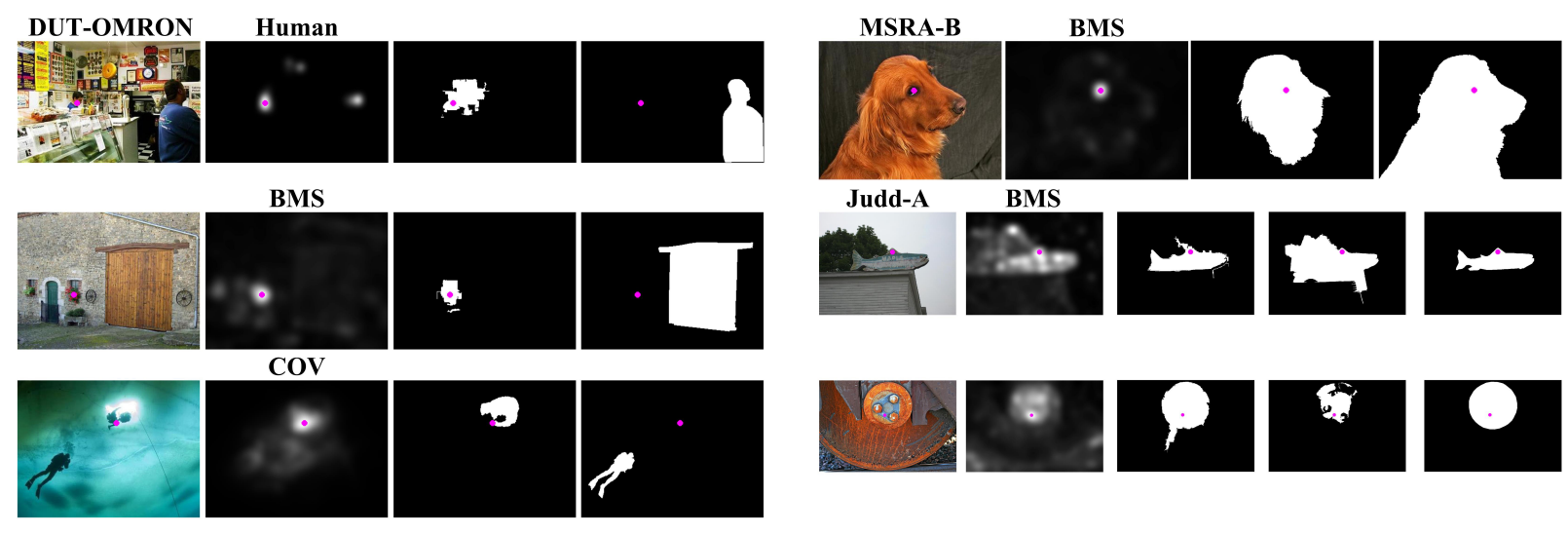

Figure 9: Examples of our methods fail. Small magenta dot represents the center of the most salient region. From left to right on DUT-OMRON and MSRA-B datasets: original image, fixation saliency map (with human or models), ours final detection, and ground truth. From left to right on Judd-A datasets: original image, fixation saliency map (with human or models), ours coarse detection, optimization result, and ground truth. Left: images from the DUTOMRON dataset where our method fails because fixations not agree with salient objects. Top right: the detection fails as salient object touches the boundary on MSRA-B. Low right: failure cases where some superpixels are incorrectly discarded/included in the optimization stage.

Table 1

Comparison of the average running time (seconds per image) on the ECSSD dataset.

\begin{tabular}{cccccccccccc}
\hline Method & MFS-COV & HDCT & RBD & GC & DRFI & MC & DSR & GMR & SF & RC & HC \\
\hline Time(s) & 1.949 & 5.541 & 0.254 & 0.028 & 18.194 & 0.184 & 3.327 & 0.653 & 0.131 & 0.087 & 0.012 \\
Code & Matlab & Matlab & Matlab & C++ & Matlab & Matlab & Matlab & Matlab & C++ & C++ & C++ \\
\hline
\end{tabular}

\subsection{Failure cases}

Failure cases of our methods to detect salient objects are shown in Fig. 9. The reasons of model failure as follows: First, fixation prediction models (i.e., human, COV, and BMS) fail to predict the most salient object in the coarse stage (e.g., the left in Fig. 9). Second, superpixels touching image boundaries are part of the salient object which is discarded in our method (the top right in Fig. 9). Third, the optimization of multilayer graph-based learning in the optimization stage is not good enough to include/discard superpixels from coarse objects (the low right in Fig. 9). The first problem indicates that human fixations not always agree with salient object and the shortcomings of fixation 


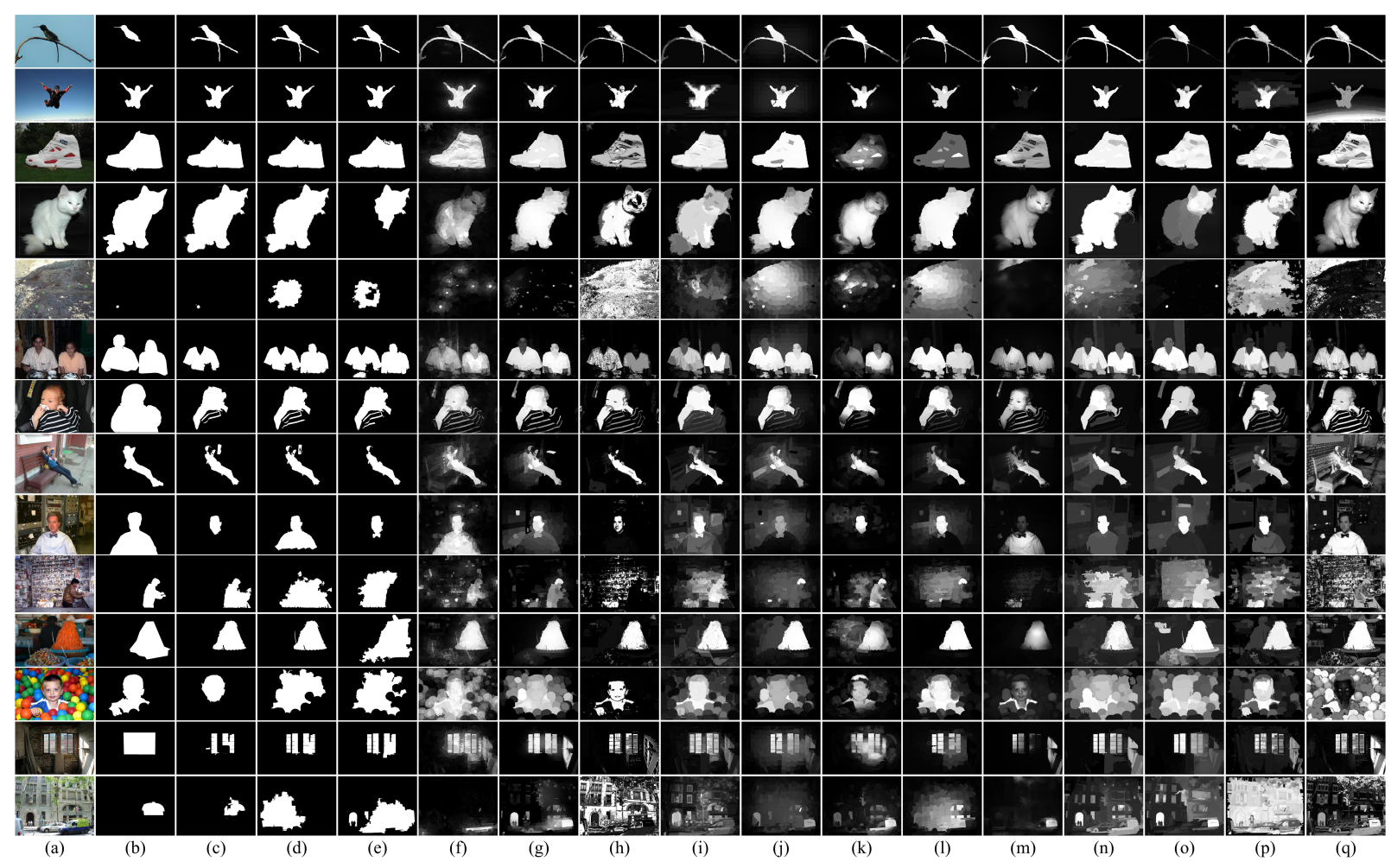

Figure 10: Comparisons of different methods on five datasets. (a) original image, (b) ground truth, (c) MFS-Human, (d) MFS-COV, (e) MFS-BMS, (f) HDCT [45], (g) RBD [49], (h) GC [50], (i) DRFI [42], (j) MC [23], (k) DSR [11], (1) GMR [24], (m) SF [47], (n) HS [29], (o) ST [48], (p) RC [46], and (q) HC [46].

prediction models. With regard to the second and third problem, modeling effort must be taken to take into account more priors of salient objects with different scales for our future work. As shown in the low right failure case of Fig. 9 , the proposed method is invalid to optimize the salient object in a scene with similar foreground/background on colors as only CIE-Lab prior is used to measure the saliency values of superpixels. In fact, all existing methods also suffer from this shortcoming, which can be addressed by introducing more priors such as depth [51], the shape of a superpixel [13] and concavity [52]. In addition, multifeature fusion is another way to cope with this shortcoming [53].

\subsection{Running time}

To further demonstrate the efficiency of our algorithm, we compute the average computational time for the proposed method MFS-COV (with COV fixation prediction) with other state-of-the-art methods mentioned above. Table 1 shows a comparison of the average running time on ECSSD dataset, whose images have average resolution of 311 $\times 375$. The top four methods are all implemented by using $\mathrm{C}++$ and our method is robust than other three methods in different scenes (see Fig. 6). Meanwhile, considering that our method is implemented by using MATLAB with unoptimized code, the computational complexity of the proposed method is comparable to that of other methods. Specifically, for MFS-COV: the coarse detection stage spends $0.332 \mathrm{~s}$ (about 17\%), and the refinement stage spends 1.617 s (about 83\%).

\section{Conclusion and future work}

In this paper, we have presented a new salient object detection method based on fixation and multilayer superpixel. First, we utilize fixation prediction models and graph-based segmentation technique to locate salient regions which are robust in cluttered scenes. It is critical to first quickly concentrate on the right regions which we only deal with in 
the subsequent processes. Second, we set different parameters for SLIC algorithm to construct three-layer superpixel maps. On single layer, we construct a shift convex hull and choose the superpixels as saliency seeds inside the convex hull. Then, we evaluate saliency of superpixels which is represented by color contrast in CIE-Lab color space by using graph-based learning. A final saliency map is generated by averaging three-layer saliency maps. We obtain the final optimization result by combining final saliency map and coarse detection region. Experimental results have shown that our method achieves comparable/better performance than the twelve state-of-the art methods on five representative benchmark datasets.

In the future research, we will consider the background prior in the coarse detection instead of discarding the superpixels touching the boundaries. As color contrast is not robust in the high-cluttered scenes, we also plan to integrate other priors other than color contrast such as shape and concavity of superpixel into the optimization process of our method. In addition, our method separates detection from segmentation which can be utilized for other purposes (e.g., human-robot interaction) by replacing the first component of our method. We are interested in introducing the current method into human-robot interaction of teleoperation. Also, it is time-consuming in the process of feature points matching for AR [54] and we will consider using the proposed method to reduce the number of feature points in the future research.

\section{Acknowledgments}

This work was supported by the Shanghai Aerospace Science and Technology Innovation Fund [grand number SAST201409] and the China National Science Foundation [grand number 71301057].

[1] X. Yao, J. Han, L. Guo, S. Bu, Z. Liu, A coarse-to-fine model for airport detection from remote sensing images using target-oriented visual saliency and crf, Neurocomputing 164 (2015) 162-172.

[2] J. Liu, Q. Sun, Sparse recognition via intra-class dictionary learning using visual saliency information, Neurocomputing 196 (2016) $70-81$.

[3] P. Peng, L. Shao, J. Han, J. Han, Saliency-aware image-to-class distances for image classification, Neurocomputing 166 (2015) $337-345$.

[4] Z. Yang, H. Xiong, Image classification based on saliency coding with category-specific codebooks, Neurocomputing 184 (2016) $188-195$.

[5] B. Schauerte, R. Stiefelhagen, "look at this!" learning to guide visual saliency in human-robot interaction, in: International Conference on Intelligent Robots and Systems, IEEE, 2014, pp. 995-1002.

[6] G. Schillaci, S. BodiroÅa, V. V. Hafner, Evaluating the effect of saliency detection and attention manipulation in human-robot interaction, International Journal of Social Robotics 5 (1) (2013) 139-152.

[7] A. Borji, M. M. Cheng, H. Jiang, J. Li, Salient object detection: A benchmark, IEEE Trans. Image Process. 24 (12) (2015) $5706-5722$.

[8] Z. S. Wang, B. X. Li, A two-stage approach to saliency detection in images, in: International Conference on Acoustics, Speech and Signal Processing, IEEE, 2008, pp. 965-968.

[9] X. Hou, L. Zhang, Saliency detection: A spectral residual approach, in: Conference on Computer Vision And Pattern Recognition, IEEE, 2007, pp. 1-8.

[10] L. Zhu, D. A. Klein, S. Frintrop, Z. Cao, A. B. Cremers, A multisize superpixel approach for salient object detection based on multivariate normal distribution estimation, IEEE Trans. Image Process. 23 (12) (2014) 5094-5107.

[11] X. H. Li, H. C. Lu, L. H. Zhang, X. Ruan, M. H. Yang, Saliency detection via dense and sparse reconstruction, in: IEEE International Conference on Computer Vision, 2013, pp. 2976-2983.

[12] A. Mishra, Y. Aloimonos, C. L. Fah, Active segmentation with fixation, in: IEEE 12th International Conference on Computer Vision, 2009, pp. 468-475.

[13] Y. Li, X. Hou, C. Koch, J. M. Rehg, A. L. Yuille, The secrets of salient object segmentation, in: IEEE Conference on Computer Vision and Pattern Recognition, 2014, pp. 280-287.

[14] A. Borji, L. Itti, State-of-the-art in visual attention modeling, IEEE Trans. Pattern Anal. Mach. Intell. 35 (1) (2013) $185-207$.

[15] A. Borji, M.-M. Cheng, H. Jiang, J. Li, Salient object detection: A survey, arXiv preprint arXiv:1411.5878 (2014).

[16] L. Itti, C. Koch, E. Niebur, A model of saliency-based visual attention for rapid scene analysis, IEEE Trans. Pattern Anal. Mach. Intell. 20 (11) (1998) 1254-1259.

[17] N. Bruce, J. Tsotsos, Saliency based on information maximization, in: Advances in neural information processing systems, 2005, pp. $155-162$.

[18] T. Judd, K. Ehinger, F. Durand, A. Torralba, Learning to predict where humans look, in: IEEE International Conference on Computer Vision, 2009, pp. 2106-2113.

[19] A. Borji, L. Itti, Exploiting local and global patch rarities for saliency detection, in: IEEE Conference on Computer Vision And Pattern Recognition, 2012, pp. 478-485.

[20] J. Zhang, S. Sclaroff, Saliency detection: A boolean map approach, in: Proceedings of the IEEE International Conference on Computer Vision, 2013, pp. 153-160.

[21] E. Erdem, A. Erdem, Visual saliency estimation by nonlinearly integrating features using region covariances, J. Vis. 13 (4) (2013) 11-11.

[22] J. Wang, H. Lu, X. Li, N. Tong, W. Liu, Saliency detection via background and foreground seed selection, Neurocomputing 152 (2015) 359-368.

[23] B. W. Jiang, L. H. Zhang, H. C. Lu, C. Yang, M. H. Yang, Saliency detection via absorbing markov chain, in: IEEE International Conference on Computer Vision, 2013, pp. 1665-1672. 
[24] C. Yang, L. H. Zhang, H. C. Lu, X. Ruan, M. H. Yang, Saliency detection via graph-based manifold ranking, in: IEEE conference on computer vision and pattern recognition, 2013, pp. 3166-3173.

[25] J. Li, Y. H. Tian, L. Y. Duan, T. J. Huang, Estimating visual saliency through single image optimization, IEEE Signal. Proc. Let. 20 (9) (2013) 845-848.

[26] K. Jiwhan, H. Dongyoon, T. Yu-Wing, K. Junmo, Salient region detection via high-dimensional color transform and local spatial support, IEEE Trans. Image Process. 25 (1) (2016) 9-23.

[27] K. Fu, C. Gong, I. Gu, J. Yang, Normalized cut-based saliency detection by adaptive multi-level region merging, IEEE Trans. Image Process. 24 (12) (2015) 5671-5683.

[28] J. Shi, J. Malik, Normalized cuts and image segmentation, IEEE Trans. Pattern Anal. Mach. Intell. 22 (8) (2000) 888-905.

[29] Q. Yan, L. Xu, J. P. Shi, J. Y. Jia, Hierarchical saliency detection, in: IEEE Conference on Computer Vision and Pattern Recognition, IEEE, 2013, pp. 1155-1162.

[30] Y. Qiu, X. Sun, M. F. She, Saliency detection using hierarchical manifold learning, Neurocomputing 168 (2015) $538-549$.

[31] A. Borji, What is a salient object? a dataset and a baseline model for salient object detection, IEEE Trans. Image Process. 24 (2) (2015) $742-56$.

[32] P. F. Felzenszwalb, D. P. Huttenlocher, Efficient graph-based image segmentation, Int. J. Comput. Vision 59 (2) (2004) 167-181.

[33] Y. C. Wei, F. Wen, W. J. Zhu, J. Sun, Geodesic saliency using background priors, in: European Conference on Computer Vision, Springer Berlin Heidelberg, 2012, pp. 29-42.

[34] T. Liu, J. Sun, N. N. Zheng, X. Tang, H. Y. Shum, Learning to detect a salient object, in: IEEE Conference on Computer Vision and Pattern Recognition, 2007, pp. 596-603.

[35] L. Vincent, P. Soille, Watersheds in digital spaces: an efficient algorithm based on immersion simulations, IEEE Trans. Pattern Anal. Mach. Intell. 13 (6) (1991) 583-598.

[36] D. Comaniciu, P. Meer, Mean shift: A robust approach toward feature space analysis, IEEE Trans. Pattern Anal. Mach. Intell. 24 (5) (2002) 603-619.

[37] A. Levinshtein, A. Stere, K. N. Kutulakos, D. J. Fleet, S. J. Dickinson, K. Siddiqi, Turbopixels: Fast superpixels using geometric flows, IEEE Trans. Pattern Anal. Mach. Intell. 31 (12) (2009) 2290-2297.

[38] R. Achanta, A. Shaji, K. Smith, A. Lucchi, P. Fua, S. Susstrunk, Slic superpixels compared to state-of-the-art superpixel methods, IEEE Trans. Pattern Anal. Mach. Intell. 34 (11) (2012) 2274-82.

[39] X. Zhu, Semi-supervised learning with graphs, Ph.D. thesis, CMU-LTI-05-192, 2005.

[40] D. Zhou, O. Bousquet, T. N. Lal, J. Weston, B. Scholkopf, Learning with local and global consistency, in: Advances in neural information processing systems, 2004, pp. 321-328.

[41] R. Achanta, S. Hemami, F. Estrada, S. Susstrunk, Frequency-tuned salient region detection, in: IEEE Conference on Computer Vision And Pattern Recognition, 2009, pp. 1597-1604.

[42] H. Z. Jiang, J. D. Wang, Z. J. Yuan, Y. Wu, N. N. Zheng, S. P. Li, Salient object detection: A discriminative regional feature integration approach, in: IEEE Conference on Computer Vision and Pattern Recognition, 2013, pp. 2083-2090.

[43] D. R. Martin, C. C. Fowlkes, J. Malik, Learning to detect natural image boundaries using local brightness, color, and texture cues, IEEE Trans. Pattern Anal. Mach. Intell. 26 (5) (2004) 530-549.

[44] M. J. Bravo, H. Farid, A scale invariant measure of clutter, J. Vis. 8 (1) (2008) 23-23.

[45] J. Kim, D. Han, Y.-W. Tai, J. Kim, Salient region detection via high-dimensional color transform, in: Proceedings of the IEEE Conference on Computer Vision and Pattern Recognition, 2014, pp. 883-890.

[46] M. M. Cheng, N. J. Mitra, X. Huang, P. H. Torr, S. M. Hu, Global contrast based salient region detection, IEEE Trans. Pattern Anal. Mach. Intell. 37 (3) (2015) 569-82.

[47] F. Perazzi, P. Krahenbuhl, Y. Pritch, A. Hornung, Saliency filters: Contrast based filtering for salient region detection, in: IEEE Conference on Computer Vision And Pattern Recognition, 2012, pp. 733-740.

[48] Z. Liu, W. Zou, O. Le Meur, Saliency tree: A novel saliency detection framework, IEEE Trans. Image Process. 23 (5) (2014) $1937-1952$.

[49] W. Zhu, S. Liang, Y. Wei, J. Sun, Saliency optimization from robust background detection, in: IEEE Conference on Computer Vision and Pattern Recognition, 2014, pp. 2814-2821.

[50] M. M. Cheng, J. Warrell, W. Y. Lin, S. Zheng, V. Vineet, N. Crook, Efficient salient region detection with soft image abstraction, in: IEEE International Conference on Computer Vision, 2013, pp. 1529-1536.

[51] H. Pan, T. Guan, Y. Luo, L. Duan, Y. Tian, L. Yi, Y. Zhao, J. Yu, Dense 3d reconstruction combining depth and rgb information, Neurocomputing 175 (2016) 644-651.

[52] Y. Lu, W. Zhang, H. Lu, X. Y. Xue, Salient object detection using concavity context, in: IEEE International Conference on Computer Vision, 2011, pp. 233-240.

[53] T. Guan, Y. Wang, L. Duan, R. Ji, On-device mobile landmark recognition using binarized descriptor with multifeature fusion, ACM Transactions on Intelligent Systems and Technology (TIST) 7 (1) (2015) 12.

[54] B. Wei, T. Guan, L. Duan, J. Yu, T. Mao, Wide area localization and tracking on camera phones for mobile augmented reality systems, Multimedia Systems 21 (4) (2015) 381-399. 